\title{
Functional and circulatory renal changes in advanced heart failure
}

\author{
Eliska Demesova ${ }^{1 *}$, Eva Goncalvesova ${ }^{2}$, Peter Slezak ${ }^{3}$, Peter Pontuch ${ }^{1}$ \\ ${ }^{1}$ St. Cyril and St. Methodius Hospital, Bratislava, Slovakia \\ ${ }^{2}$ National Cardiovascular Institute, Bratislava, Slovakia \\ ${ }^{3}$ Institute of Simulation and Virtual Medical Education, Faculty of Medicine, Comenius University, \\ Bratislava, Slovakia
}

Objectives: The aim of the study was to describe the rela-
tions between heart and renal functions and to investigate
whether reduced glomerular filtration rate is influenced ra-
ther by reduced perfusion or venous congestion.

Patients and Methods: A prospective cohort study of 101 patients (69 men, 32 women) with chronic heart failure aged 52 (median age). Patients included in the study consist of two distinct groups. The group of patients with advanced chronic heart failure on the waiting list for orthotopic heart transplantation $(\mathrm{N}=78)$ and the group of patients with pulmonary arterial hypertension $(\mathrm{N}=23)$. The etiology of chronic heart failure in the first group was in $57 \%$ ischemic and in $43 \%$ non-ischemic. In the whole cohort, $23.7 \%$ of patients had diabetes mellitus, $30 \%$ were obese and $42.6 \%$ had arterial hypertension. We analyzed the blood samples, parameters of echocardiography and right heart catheterization.

Results: Left ventricular ejection fraction correlated with the estimated glomerular filtration rate eGFR $(r=0.214, p=$ 0.036 ) in the complete set of patients, but not in the two divided groups. There was no correlation between cardiac output and eGFR in either group. We found a correlation

\section{Received: $28^{\text {th }}$ Apr 2014}

*Address for correspondence: 4th Department of Internal Medicine, St. Cyril and St. Methodius Hospital, Antolska 11, 85107 Bratislava, Slovakia.

Phone: +421905951113

E-mail: eli.demesova@gmail.com between cardiac output and renal perfusion pressure in a complete set $(r=0.232, p=0.0225)$ and in patients with chronic heart failure $(r=0.254, p=0.0278)$, but no significant correlation in patients with pulmonary hypertension. There were no significant correlations between central venous pressure and eGFR in any of the studied groups. Echocardiographic monitoring of the right heart sections showed a correlation between TAPSE as a marker of right ventricular function and eGFR in the complete set $(r=$ $0.351, p=0.0033$ ) and in patients with chronic heart failure $(r=0.417, p=0.0039)$, but not in patients with pulmonary hypertension. In the complete set, the mean pulmonary artery pressure (PAP) correlated with the variables determining the renal function: PAP and renal perfusion pressure $(r$ $=-0.345, p=0.002)$. PAP and eGFR $(r=-0.299, p=0.009)$. In the other two studied groups, these correlations were not significant.

Conclusion: In the selected group of heart failure patients, we found left and right ventricular functions to be the main determinants for renal function. Current cardiac output or right atrial pressure as the markers of renal perfusion was not associated with renal functions in advanced, but stable heart failure patients with low burden of extracardiac comorbidities.

KEYWORDS: renal venous congestion, cardiac output, renal perfusion, cardiorenal syndrome, advanced heart failure.

CITATION: Cardio Croat. 2014;9(5-6):226.

\section{Literature}

1. Guglin M, Rivero A, Matar F, Garcia M. Renal dysfunction in heart failure is due to congestion but not low output. Clin Cardiol. 2011;34(2):113-6.

2. Ronco C, Haapio M, House AA, Anavekar N, Bellomo R. Cardiorenal syndrome. J Am Coll Cardiol. 2008;52(19):1527-39.

3. Jaap A, Bongartz L, Gaillard C, Braam B. Renal venous congestion and renal function in congestive heart failure. J Am Coll Cardiol. 2009;54(17):1632 